\title{
Complete field descriptions in three-dimensional multiple scattering problems: a transfer-matrix approach
}

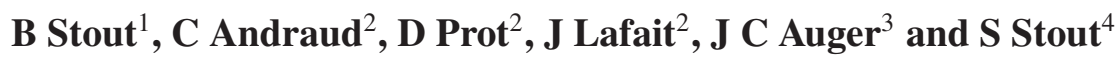 \\ ${ }^{1}$ Institut Fresnel, UMR 6133, Case 161, Faculté des Sciences et Techniques, \\ Centre de Saint Jérome, 13397 Marseille Cedex 20, France \\ ${ }^{2}$ Laboratoire d'Optique des Solides de l'Université Pierre et Marie Curie, UMR 7601, Case 80, \\ 4 place Jussieu, 75252 Paris Cedex 05, France \\ ${ }^{3}$ Centro de Investigacion en Polimeros, COMEX, Marcos Achar Logaton, 55885 Tepexpan, \\ Estado de México 2, Mexico \\ ${ }^{4}$ Laboratoire d'Interfaces Physique-Medecine-Biologie, Faculté des Sciences et Techniques, \\ Centre de Saint Jérome, 13397 Marseille Cedex 20, France
}

Received 29 January 2002, in final form 17 May 2002

Published 5 September 2002

Online at stacks.iop.org/JOptA/4/S182

\begin{abstract}
We illustrate that a recently formulated recursive transfer matrix method can be used to reliably calculate the electromagnetic fields throughout three-dimensional systems of strongly scattering spheres, and/or coated spheres. The exceptional features of our technique are its particularly stable and reliable numeric implementations. In this work, we present new self-consistent formulae which permit the verification of the numerical stability at any stage of the calculations, and which ensure the satisfaction of the underlying multiple scattering equations for an arbitrary incident wave.
\end{abstract}

Keywords: Multiple scattering, field fluctuations, Mie scattering

(Some figures in this article are in colour only in the electronic version)

Exact solutions to finite multiple scattering systems can provide important insights into the numerous approximate methods that are typically used to describe multiple scattering. Perhaps even more importantly, exact solutions are a good means of studying field fluctuations in multiple scattering systems.

Invoking the addition theorem [1, 2], one can rather readily describe a multiple scattering system via coupled matrix equations acting on a spherical wave development of the incident field [3-7]. After a suitable truncation, the complete solution to this problem can then, in principle, be obtained by the inversion of an entire 'system' matrix [3, 5-7]. Analogous problems for two-dimensional systems of infinite cylinders have been treated with success in this manner $[8,9]$. The enormous number of unknowns and the sparse nature of the matrix, however, often prevents a direct matrix inversion solution for three-dimensional systems of spheres [5, 6, 10-12].

Several authors have proposed the calculation of cluster transfer matrices, $T_{N}^{c l}$, via recursive approaches which build the transfer matrix one particle at a time [11,13]. A major obstacle in these recursive approaches is that they suffer from numerical instabilities associated with partial-wave space truncations as recently discussed by Siqueira and Sarabandi [12], and by us [5]. Another deficiency of these new transfer-matrix approaches is that in practice, one often loses (or discards) local field information. In response to these difficulties, we have recently developed and applied a modified recursive technique which eliminates the aforementioned numerical difficulties [3$5]$.

In this work, we illustrate that this new recursive transfermatrix technique can also be used to calculate the field distribution throughout multiple scattering systems (both near and far field). Since the numerical stability of the recursive transfer methods is still questioned in the multiple scattering community, we introduce here new self-consistence formulae which must be satisfied by our transfer matrices. We illustrate here that the satisfaction of these relations implies the satisfaction of the underlying multiple scattering equations for arbitrary incident wave configurations. To date, we have found that these relations are satisfied by our recursive matrix transfer matrices up to computer precision round off. 


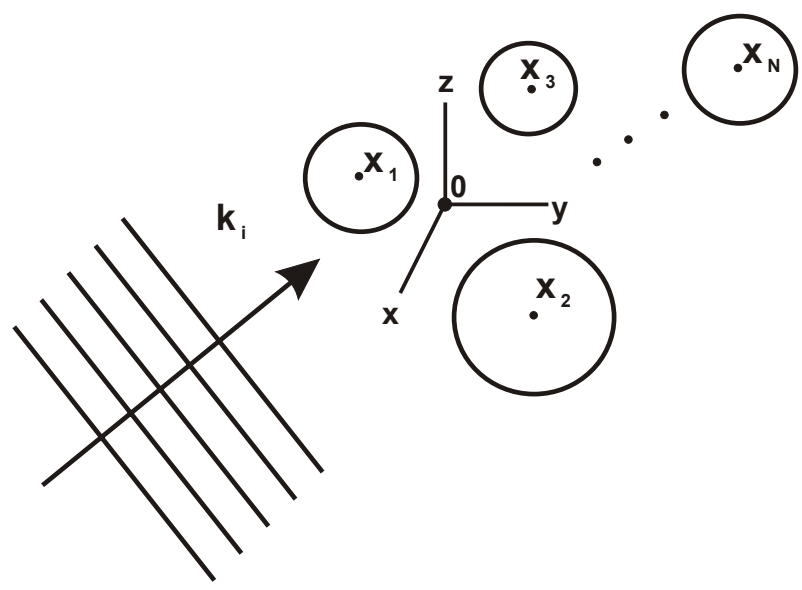

Figure 1. A multiple scattering system of spheres in the presence of an incident field. The centres of the spheres are denoted $\boldsymbol{x}_{j}$, $j=1, \ldots, N, N$ being the number of spheres in the system.

The outline of this work is as follows. Section 1.1 gives a brief introduction to our notation, and the formulation of the solution of the multiple scattering problem in terms of the body-centred transfer matrices, $T_{N}^{(i, j)}$. In section 1.2 , we present a recently derived and numerically stable algorithm for calculating $T_{N}^{(i, j)}$. We then present in section 1.3, new self-consistence formulae for testing the numeric stability of our recursive technique. Formulae for extracting the electric field distribution are given in section 2. We also present in this section, some illustrative results for field distributions in threedimensional systems exhibiting multiple dependent scattering.

\section{Spherical wave expansions and transfer-matrix constructions}

\subsection{Spherical wave expansions}

A field, $\boldsymbol{E}_{i}$, incident on an $N$-particle system can be developed in terms of regular spherical partial waves developed about some point $\mathbf{0}$ chosen as the origin (see figure 1)

$$
\begin{aligned}
& \boldsymbol{E}_{i}(\boldsymbol{r})=E \sum_{n=1}^{\infty} \sum_{m=-n}^{n}\left(\mathcal{R} g\left\{\boldsymbol{M}_{n m}(k \boldsymbol{r})\right\} a_{n m}^{M}+\mathcal{R} g\left\{\boldsymbol{N}_{n m}(k \boldsymbol{r})\right\} a_{n m}^{N}\right) \\
& =E[\mathcal{R} g\{\boldsymbol{M}(k \boldsymbol{r})\}, \mathcal{R} g\{\boldsymbol{N}(k \boldsymbol{r})\}] \cdot\left[\begin{array}{c}
a^{M} \\
a^{N}
\end{array}\right] \\
& \equiv E \mathcal{R} g\left\{\mathcal{E}^{t}(k \boldsymbol{r})\right\} \cdot a
\end{aligned}
$$

where $\boldsymbol{M}_{n m}$ and $\boldsymbol{N}_{n m}$ are the vector spherical waves (see the appendix), and $E$ is a real parameter which determines the amplitude of the wave. In (1) we introduce a compact matrix notation $[11,14]$ in which the column vector ' $a$ ' is composed of the incident field coefficients while $\mathcal{E}(k \boldsymbol{r})$ is as an abstract vector composed of the vector wavefunctions $M(k r)$ and $\boldsymbol{N}(k \boldsymbol{r})$. The notation $\mathcal{R} g\{\}$ stands for 'the regular part of' [14] (see the appendix).

In a multiple scattering system of spheres, or coated spheres, the total field, $\boldsymbol{E}_{t}(\boldsymbol{r})$, at a point exterior to all the scatterers can be written as the sum of the incident field, and a set of 'individual' scattered fields centred respectively on each of the particle centres

$$
\boldsymbol{E}_{t}(\boldsymbol{r})=\boldsymbol{E}_{i}+\sum_{j=1}^{N} \boldsymbol{E}_{s}^{(j)}\left(\boldsymbol{r}_{j}\right)=\boldsymbol{E}_{i}+E \sum_{j=1}^{N} \mathcal{E}^{t}\left(k \boldsymbol{r}_{j}\right) \cdot f^{(j)}
$$

The centre of the $j$ th particle is denoted $\boldsymbol{x}_{j}$, and the spherical coordinates relative to each scatterer are $\boldsymbol{r}_{j} \equiv \boldsymbol{r}-\boldsymbol{x}_{j}$. The scattered field emanating from the $j$ th particle, $\boldsymbol{E}_{s}^{(j)}$, can be developed in terms of outgoing spherical waves (see (19) in the appendix). The coefficients of the scattered field partial-wave developments are collected into respective scattering 'vectors', $f^{(j)}$.

The solution to a multiple $N$-scatterer system can be compactly expressed in terms of the body-centred transfer matrices, $T_{N}^{(j, k)}[5,6]$

$$
f^{(j)}=\sum_{l=1}^{N} T_{N}^{(j, l)} \cdot \beta^{(l, 0)} \cdot a
$$

where $\beta^{(l, 0)} \equiv \beta\left(k \boldsymbol{x}_{l}\right)$ is the regular translation-addition matrix (see the appendix). The coefficient vector ' $\beta^{(l, 0)} \cdot a$ ' is then the field expansion of the incident field in a spherical wave basis developed around the centre of $l$ th scatterer, $\boldsymbol{x}_{l}$.

\subsection{Transfer-matrix constructions}

The building blocks for calculating $T_{N}^{(j, k)}$ are the individual transfer matrices of the scatterers taken in isolation, $T_{1}^{(j)}$, and the irregular translation-addition matrices, $\alpha^{(j, k)}$ (see the appendix). For a sphere, $T_{1}^{(j)}$ is a diagonal matrix whose elements are given by the Mie coefficients $[15,16]$.

Our recursive prescription for calculating $T_{N}^{(j, k)}$ is to add the scatterers one at a time to the system in an arbitrary order. Assuming that the $T_{N-1}^{(k, j)}$ matrices for an $N-1$ particle system have been calculated, the $N$-particle matrix, $T_{N}^{(N, N)}$, for a particle $N$ added to the system is calculated by a matrix inversion carried out in a spherical-wave space describing a single particle $[3,5]$ :

$$
T_{N}^{(N, N)}=T_{1}^{(N)} \cdot\left[\mathrm{I}-\sum_{j, k=1}^{N-1} \alpha^{(N, k)} \cdot T_{N-1}^{(k, j)} \cdot \alpha^{(j, N)} \cdot T_{1}^{(N)}\right]^{-1} .
$$

Once this matrix is calculated, the $T_{N}^{(j, k)}$ matrices involving the $N$ th particle can be obtained by matrix multiplications

$$
\begin{array}{ll}
T_{N}^{(N, k)}=T_{N}^{(N, N)} \cdot \sum_{i=1}^{N-1} \alpha^{(N, i)} \cdot T_{N-1}^{(i, k)} & k \neq N \\
T_{N}^{(j, N)}=\sum_{i=1}^{N-1} T_{N-1}^{(j, i)} \cdot \alpha^{(i, N)} \cdot T_{N}^{(N, N)} & j \neq N .
\end{array}
$$

The readjustment of $T_{N-1}^{(j, k)} ; j \neq N, k \neq N$ so that they become $N$-body scatterer centred matrices, $T_{N}^{(j, k)}$ is also achieved by matrix multiplication

$$
T_{N}^{(j, k)}=T_{N-1}^{(j, k)}+\sum_{i=1}^{N-1} T_{N-1}^{(j, i)} \cdot \alpha^{(i, N)} \cdot T_{N}^{(N, k)} \quad j, k \neq N .
$$


It is worth noting that although the number of $T_{N}^{(j, k)}$ matrices grows as $N^{2}$, each of these matrices can be reliably truncated following the usual Wiscomb criteria for isolated particles. Our recursive method is not subject to the numeric cut-off instabilities which plague the other recursive techniques known to us.

\subsection{Self-consistence relations}

Returning to the basic multiple scattering equations [5], and invoking the definition of the $N$-body transfer matrices, (3), one can show that $T_{N}^{(j, k)}$ satisfies

$$
\begin{gathered}
T_{N}^{(j, j)}=T_{1}^{(j)}+T_{1}^{(j)} \cdot \sum_{l=1, l \neq j}^{N} \alpha^{(j, l)} \cdot T_{N}^{(l, j)} \\
T_{N}^{(j, k)}=T_{1}^{(j)} \cdot \sum_{l=1, l \neq j}^{N} \alpha^{(j, l)} \cdot T_{N}^{(l, k)} \quad k \neq j .
\end{gathered}
$$

In the relatively low particle number systems that we have studied so far, these relations are always satisfied up to the numerical precision adopted for the calculations. This property holds true only because of the high numeric stability of our recursive formulation. Nevertheless, the iteration of (8) may in the future prove useful in the elimination of cumulative roundoff errors in large systems.

We shall now verify that the satisfaction of (8) implies a full solution of the multiple scattering equation. Multiplying the RHS of $(8 a)$ and $(8 b)$, respectively, by $\beta^{(j, 0)}$ and $\beta^{(k, 0)}$, adding the resulting equations, and finally summing over all $k \neq j$, we obtain

$$
\sum_{k=1}^{N} T_{N}^{(j, k)} \cdot \beta^{(k, 0)}=T_{1}^{(j)} \cdot \beta^{(j, 0)}+T_{1}^{(j)} \cdot \sum_{l \neq j, k}^{N} \alpha^{(j, l)} \cdot T_{N}^{(l, k)} \cdot \beta^{(k, 0)} .
$$

Multiplying this equation from the right by the coefficients ' $a$ ' of an arbitrary incident field and using the expression for the scattering vectors, (3), one finds that (9) then takes the form of the fundamental multiple scattering equation $[5,11]$

$$
f^{(j)}=T_{1}^{(j)} \cdot \beta^{(j, 0)} \cdot a+T_{1}^{(j)} \cdot \sum_{l \neq j}^{N} \alpha^{(j, l)} \cdot f^{(l)} .
$$

\section{Field distributions}

\subsection{Incident field}

An incident wave of particular utility is the homogenous plane wave, $\boldsymbol{E}_{i}^{p l}$, described via a wavevector, $\boldsymbol{k}_{i}$, and a transverse polarization vector, $\hat{\boldsymbol{e}}_{\gamma}(\gamma=1,2$ in a suitable basis $)$. The coefficient vector for this special incident field is denoted $p_{\gamma}$ (see (18) of the appendix)

$$
\boldsymbol{E}_{i}^{p l}(\boldsymbol{r})=E \exp \left(\mathrm{i} \boldsymbol{k}_{i} \bullet \boldsymbol{r}\right) \hat{\boldsymbol{e}}_{\gamma} \equiv E \mathcal{R} g\left\{\mathcal{E}^{t}(k \boldsymbol{r})\right\} \cdot p_{\gamma},
$$

where - denotes a dyadic scalar product, and $p_{\gamma}$ are eigenvectors of the regular translation matrices [4], i.e. $\beta(k \boldsymbol{x})$. $p_{\gamma}=\mathrm{e}^{\mathrm{i} k_{i} \cdot x} p_{\gamma}$. This 'phase' relation alleviates potential truncation problems associated with the regular translationaddition matrices [4]. Applying this relation to (3), the scattering coefficients $f^{(l)}$ in the presence of an incident plane wave can be written

$$
f^{(j)}=\sum_{k=1}^{N} T_{N}^{(j, k)} \cdot \beta^{(k, 0)} \cdot p_{\gamma}=\sum_{k=1}^{N} \mathrm{e}^{\mathrm{i} k_{i} \cdot x_{k}} T_{N}^{(j, k)} \cdot p_{\gamma} .
$$

\subsection{Total field formulae}

Inserting (12) into (2), the total electric field in a multiple scattering system for an incident plane wave is

$$
\begin{aligned}
\boldsymbol{E}_{t}(\boldsymbol{r}) & =E\left[\exp \left(\mathrm{i} \boldsymbol{k}_{i} \bullet \boldsymbol{r}\right) \hat{\boldsymbol{e}}_{\gamma}\right. \\
& \left.+\sum_{j=1, k=1}^{N} \mathrm{e}^{\mathrm{i} \boldsymbol{k}_{i} \cdot \boldsymbol{x}_{k}} \overline{\bar{C}}\left(\hat{\boldsymbol{r}}_{j}\right) \bullet \mathcal{E}^{t}\left(k \boldsymbol{r}_{j}\right) \cdot T_{N}^{(j, k)} \cdot p_{\gamma}\right]
\end{aligned}
$$

where $\overline{\bar{C}}\left(\hat{\boldsymbol{r}}_{j}\right)$ is the dyadic which transforms vectors in the $\hat{\boldsymbol{r}}_{j}$ coordinate basis into the Cartesian coordinate system

$$
\overline{\bar{C}}\left(\hat{\boldsymbol{r}}_{j}\right)=\left(\begin{array}{ccc}
\sin \theta_{j} \cos \phi_{j} & \cos \phi_{j} \cos \theta_{j} & -\sin \phi_{j} \\
\sin \theta_{j} \sin \phi_{j} & \sin \phi_{j} \cos \theta_{j} & \cos \phi_{j} \\
\cos \theta_{j} & -\sin \theta_{j} & 0
\end{array}\right) .
$$

The total electric field in the interior of a particle $j$ in an $N$-particle cluster, $\boldsymbol{E}_{I}^{(j)}(\boldsymbol{r})$, is described via the internal field coefficients $i^{(j)}, \boldsymbol{E}_{I}^{(j)}(\boldsymbol{r})=E \mathcal{R} g\left\{\mathcal{E}^{t}\left(k \boldsymbol{r}_{j}\right)\right\} \cdot i^{(j)}$. For spherical particles, the boundary conditions at the surface of the particle impose that these $i^{(j)}$ coefficients are related to the scattering coefficients, $f^{(j)}$ via the relation $[4,15,16]$

$$
\begin{gathered}
i^{(j)}=\Lambda^{(j)} \cdot f^{(j)}=\sum_{k=1}^{N} \Lambda^{(j)} \cdot T_{N}^{(j, k)} \cdot \beta^{(k, 0)} \cdot a \\
\Lambda^{(j)}=\left[\begin{array}{cc}
\Lambda^{M,(j)} & 0 \\
0 & \Lambda^{N,(j)}
\end{array}\right]
\end{gathered}
$$

where the $\Lambda^{A,(j)}$ are diagonal matrices

$$
\begin{aligned}
\Lambda_{n m, v \mu}^{M,(j)} & =\delta_{n v} \delta_{m \mu} \frac{\mathrm{i} \mu_{j} \rho_{j}}{\mu \rho_{j} \psi_{n}^{\prime}\left(\rho_{j} \chi_{j}\right) \psi_{n}\left(\chi_{j}\right)-\mu_{j} \psi_{n}\left(\rho_{j} \chi_{j}\right) \psi_{n}^{\prime}\left(\chi_{j}\right)} \\
\Lambda_{n m, v \mu}^{N,(j)} & =\delta_{n v} \delta_{m \mu} \frac{\mathrm{i} \mu_{j} \rho_{j}}{\mu_{j} \psi_{n}\left(\chi_{j}\right) \psi_{n}^{\prime}\left(\rho_{j} \chi_{j}\right)-\mu \rho_{j} \psi_{n}\left(\rho_{j} \chi_{j}\right) \psi_{n}^{\prime}\left(\chi_{j}\right)} .
\end{aligned}
$$

The functions $\psi_{n}(x)$ are Ricatti spherical Bessel functions, $\psi_{n}(x) \equiv x j_{n}(x)$, and $\rho_{j} \equiv \sqrt{\frac{\varepsilon_{j} \mu_{j}}{\varepsilon \mu}}$ where $\varepsilon$ and $\mu$ are the constitutive parameters of the host medium.

\subsection{Demonstrative calculations}

We now demonstrate an application of the formalism elaborated in this work to the problem of the field distribution in a system exhibiting strong dependent scattering. We consider systems of seven identical dielectric scatterers of refraction index $n_{s} \simeq 2.5$ (approximately that of $\mathrm{TiO}_{2}$ ) in a dielectric host medium of index $n \simeq 1.5$ (corresponding to common polymers). One sphere is placed at the origin of the coordinate system. Pairs of spheres are placed equidistantly on either side of the central sphere along each of the $\hat{\boldsymbol{x}}, \hat{\boldsymbol{y}}$, and $\hat{\boldsymbol{z}}$ axis. We take a size parameter corresponding to strong scattering, 

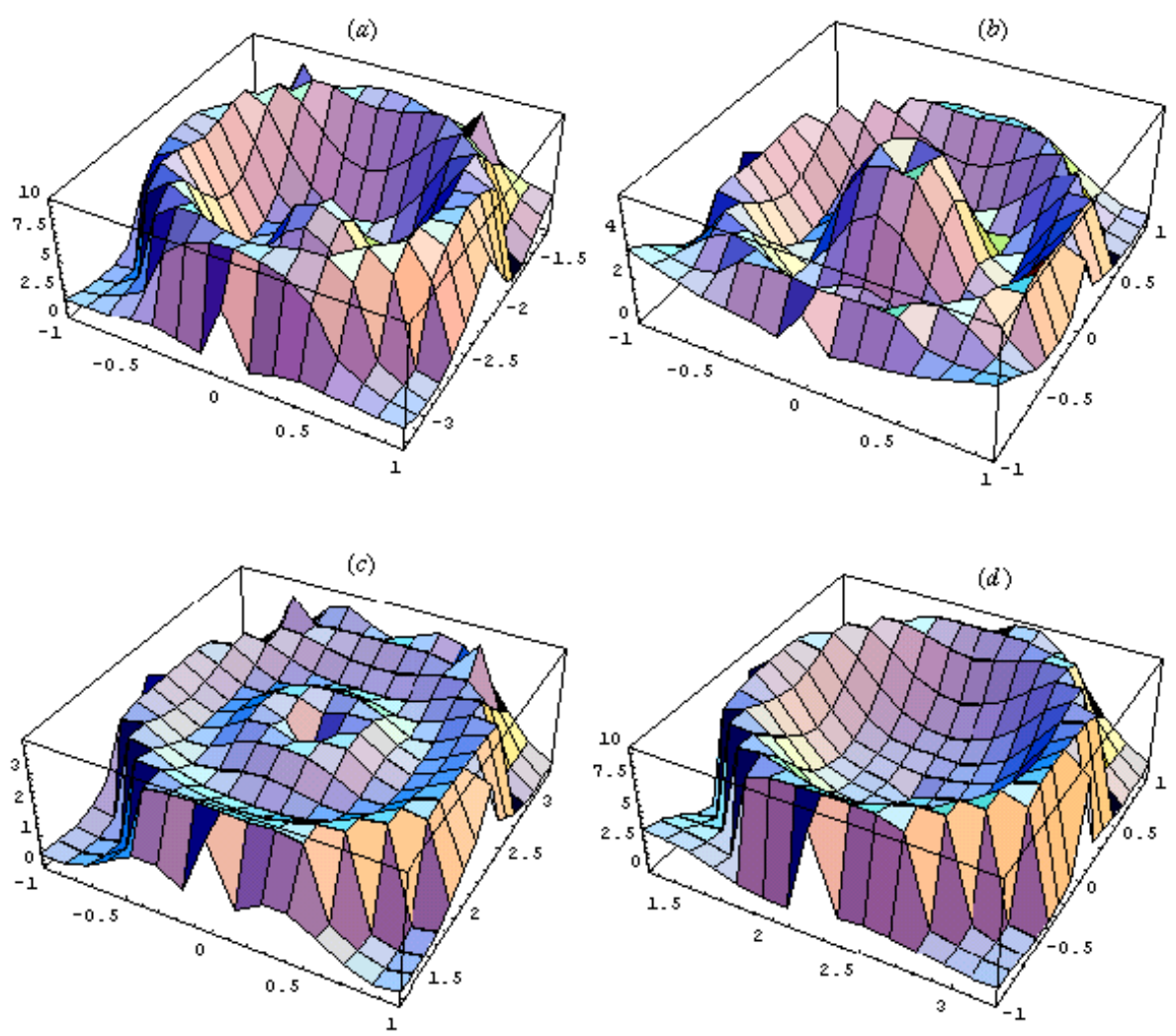

Figure 2. Field distributions within the spheres in the seven-particle multiple scattering system described in the text. The distances are scaled so that $R=1$. The normalized square field distributions, $\boldsymbol{E}_{t}^{2}(\boldsymbol{r}) / E^{2}$, are plotted in the $\hat{\boldsymbol{y}}-\hat{\boldsymbol{z}}$ plane. Plots $(a),(b)$ and $(c)$ contain, respectively, the three particles lying along the $\hat{z}$ axis, centred respectively on $\{0,0,-R\},\{0,0,0\}$ and $\{0,0, R\}$. Part $(d)$ plots the field within the particle centred on $\{0, R, 0\}$. The other $\hat{y}$ axis particle has a symmetric field distribution. The fields in the two outer particles lying along the $\hat{x}$ axis are not shown since they lie outside the plane being considered.

$\chi \equiv \frac{2 \pi R}{\lambda}=3$, where $\lambda$ is the wavelength in the host media, and $R$ is the radius of the spheres. The centre of each of the six 'exterior' spheres is placed at a distance of $2.25 R$ from the origin. One can therefore expect rather strong dependent scattering effects. We found that a partial-wave truncation of $N_{\max }=4$ truncation is already sufficient to obtain $\sim 99.9 \%$ of the total cross section of this system.

Using the cross section formulae of [4], applied to the above configuration with an incident plane wave polarized along the $\hat{\boldsymbol{x}}$ (or $\hat{\boldsymbol{y}}$ ) axis, the total cross section 'efficiency' factor per sphere is, $Q \equiv \sigma_{\text {tot }} /\left(7 \times \pi R^{2}\right) \simeq 2.71$. This shows that despite the proximity of the spheres, and the fact that certain spheres in this configuration are strongly 'shadowed', the strong interaction with the light continues in a manner such that the spheres scatter considerably more light than even their (independent) geometric cross sections would suggest. Although the present system is relatively symmetric, the cross section has noticeable variations with respect to both orientation and polarization. Analytical orientation averages can be performed using the 'trace' formulae of $[5,6]$. In the present case, the orientation average of the total efficiency factor is $\langle Q\rangle_{o} \equiv\left\langle\sigma_{\text {tot }}\right\rangle_{o} /\left(7 \times \pi R^{2}\right) \simeq 2.85$. The transfer matrices of our calculation satisfy the self-consistent formulae of (8) up to computer round-off errors $\left(\sim 10^{-18}\right)$ and as shown in section 1.3 , this condition imposes the satisfaction of the underlying multiple scattering equations involving the scattering coefficients.
We have not found a satisfactory way to graphically represent the full vector field information in a threedimensional system. We will therefore choose to plot $\left|\boldsymbol{E}_{t}^{(j)}(\boldsymbol{r})\right|^{2} / E^{2}$ along a plane section of the system. The incident wavevector, is chosen to be parallel to the positive $\hat{z}$ axis, $\boldsymbol{k}_{i}=\hat{\boldsymbol{z}}$ with a linear polarization along the $\hat{\boldsymbol{y}}$ axis. In order to highlight the regions of most interest, we present field maps inside and near the particles. In figure 2, we present the maps of $\left|\boldsymbol{E}_{t}^{(j)}(\boldsymbol{r})\right|^{2} / E^{2}$ in the $\hat{\boldsymbol{y}}-\hat{\boldsymbol{z}}$ plane of the system within a square of $y \in\left\{-R+y_{j}, R+y_{j}\right\}, z \in\left\{-R+z_{j}, R+z_{j}\right\}$ around the respective particle centres, $\boldsymbol{x}_{j} \rightarrow\left\{x_{j}, y_{j}, z_{j}\right\}$. These figures show the importance of dependant scattering effects on the field within the scatterers. In the regions outside the spheres, one finds some rather small regions containing rather large field enhancements. As one might expect from the symmetry of the system, the largest of these 'hot spots' lie along the $z$ axis, and we illustrate some of the most notable of these in figure 3 . The largest of these peaks contain squared field amplitudes that are more than eight times larger than that of the incident field.

\section{Conclusions}

Once the transfer matrix of a system has been calculated, and its numerical accuracy tested via (8), one can rather easily study the field distributions for arbitrary incident wave configurations and polarizations using the formulae of section 2 . The 

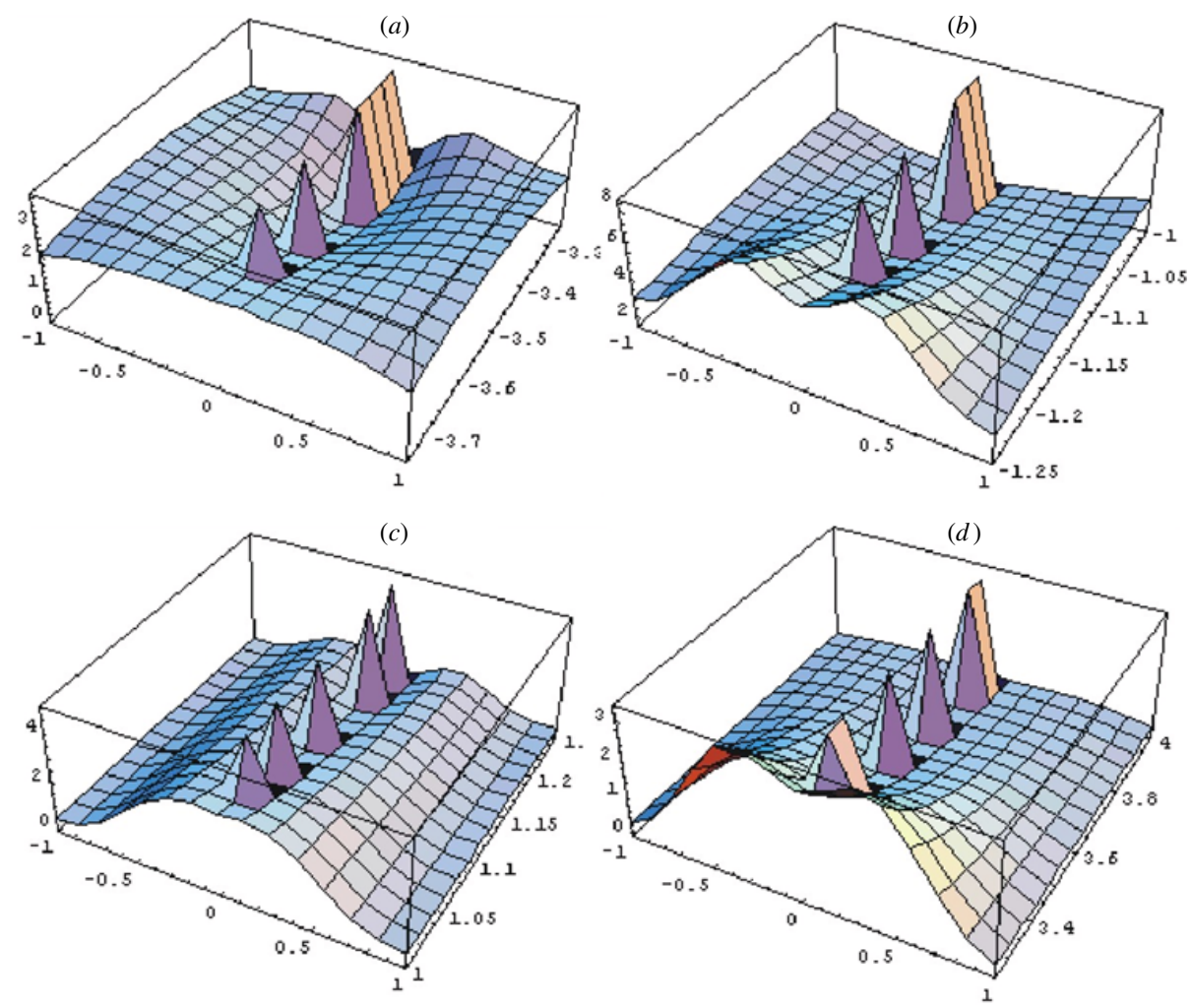

Figure 3. The field distributions of some of the principle 'hot spots' exterior to the particles. Due to the symmetry of the problem, these lie principally along the $\hat{z}$ axis. The four zones illustrated are: $(a)$ in front of the system; $(b)$ between the particles of figures $2(a)$ and $(b)$; $(c)$ between the particles of figures $2(b)$ and $(d)$; and $(d)$ behind the system. The terms in front and behind are determined with respect to the incident wave.

field maps can yield valuable information concerning field fluctuations in multiple scattering systems. More elaborate studies with larger numbers of spheres and/or coated spheres (not necessarily concentric) are currently underway.

\section{Appendix. Spherical waves and vector spherical harmonics}

The three normalized vector spherical harmonics (VSHs) used by us, can be explicitly written in terms of the associated Legendre functions

$$
\begin{gathered}
\boldsymbol{Y}_{n m}(\hat{\boldsymbol{r}})=\gamma_{n m} \sqrt{n(n+1)} P_{n}^{m}(\cos \theta) \mathrm{e}^{\mathrm{i} m \phi} \hat{\boldsymbol{r}}=Y_{n m}(\theta, \phi) \hat{\boldsymbol{r}} \\
\boldsymbol{X}_{n m}(\hat{\boldsymbol{r}})=\gamma_{n m}\left[-\frac{\mathrm{i} m}{\sin \theta} P_{n}^{m}(\cos \theta) \mathrm{e}^{\mathrm{i} m \phi} \hat{\theta}+\frac{\mathrm{d}}{\mathrm{d} \theta} P_{n}^{m}(\cos \theta) \mathrm{e}^{\mathrm{i} m \phi} \hat{\phi}\right] \\
\boldsymbol{Z}_{n m}(\hat{\boldsymbol{r}})=\gamma_{n m}\left[\frac{\mathrm{d}}{\mathrm{d} \theta} P_{n}^{m}(\cos \theta) \mathrm{e}^{\mathrm{i} m \phi} \hat{\theta}+\frac{\mathrm{i} m}{\sin \theta} P_{n}^{m}(\cos \theta) \mathrm{e}^{\mathrm{i} m \phi} \hat{\phi}\right] \\
\gamma_{n m} \equiv \sqrt{\frac{(2 n+1)(n-m) !}{4 \pi n(n+1)(n+m) !}} .
\end{gathered}
$$

The coefficients of an incident plane wave can be compactly expressed as ordinary (three) vector scalar products between $\hat{\boldsymbol{e}}_{\gamma}$ and the transverse VSHs

$$
\begin{gathered}
{\left[p_{\gamma}\right]_{n m}^{M}=-i^{n} 4 \pi \boldsymbol{X}_{n m}^{*}\left(\hat{\boldsymbol{k}}_{i}\right) \bullet \hat{\boldsymbol{e}}_{\gamma}} \\
{\left[p_{\gamma}\right]_{n m}^{N}=-i^{n+1} 4 \pi \boldsymbol{Z}_{n m}^{*}\left(\hat{\boldsymbol{k}}_{i}\right) \bullet \hat{\boldsymbol{e}}_{\gamma} .}
\end{gathered}
$$

The (outgoing) normalized vector spherical waves can be expressed in terms of VSHs and spherical Hankel functions of the first kind, $h_{n}(k r)$

$$
\begin{aligned}
\boldsymbol{M}_{n m}(k \boldsymbol{r}) & \equiv-h_{n}(k r) \boldsymbol{X}_{n m}(\hat{\boldsymbol{r}}) \\
\boldsymbol{N}_{n m}(k \boldsymbol{r}) & \equiv \frac{1}{k r}\left\{\sqrt{n(n+1)} h_{n}(k r) \boldsymbol{Y}_{n m}(\hat{\boldsymbol{r}})\right. \\
+ & {\left.\left[k r h_{n}(k r)\right]^{\prime} \boldsymbol{Z}_{n m}(\hat{\boldsymbol{r}})\right\} }
\end{aligned}
$$

Regular spherical waves are denoted $\mathcal{R} g\{\boldsymbol{M}\}$ and $\mathcal{R} g\{\boldsymbol{N}\}$, and obtained by replacing the $h_{n}(x)$ with spherical Bessel functions, $j_{n}(x)$.

The vector wave addition theorem permits the expression of spherical waves expressed in coordinates $r=O P$ in terms of spherical waves expressed around a different origin, $\boldsymbol{r}^{\prime}=\boldsymbol{O}^{\prime} \boldsymbol{P}[1,2,11,14]$

$$
\begin{array}{cl}
\mathcal{E}^{t}(k \boldsymbol{r})=\mathcal{E}^{t}\left(k \boldsymbol{r}^{\prime}\right) \cdot \beta\left(k \boldsymbol{r}_{0}\right) & r^{\prime}>r_{0} \\
\mathcal{E}^{t}(k \boldsymbol{r})=\mathcal{R} g\left\{\mathcal{E}^{t}\left(k \boldsymbol{r}^{\prime}\right)\right\} \cdot \alpha\left(k \boldsymbol{r}_{0}\right) & r^{\prime}<r_{0} \\
\mathcal{R} g\left\{\mathcal{E}^{t}(k \boldsymbol{r})\right\}=\mathcal{R} g\left\{\mathcal{E}^{t}\left(k \boldsymbol{r}^{\prime}\right)\right\} \cdot \beta\left(k \boldsymbol{r}_{0}\right) & \forall|\boldsymbol{r}|
\end{array}
$$

where $\boldsymbol{r}_{0} \equiv \boldsymbol{O} \boldsymbol{O}^{\prime}$. The $\alpha\left(k \boldsymbol{r}_{0}\right)$ and $\beta\left(k \boldsymbol{r}_{0}\right)$ are the irregular and regular translation-addition matrices in the normalized notation, respectively [14]. We have used the notation, $\alpha, \beta$, for these matrices as adopted by Chew for scalar fields [11]. In the notation of Mackowski [6] these matrices are $H\left(k r_{0}\right)$ and $J\left(k \boldsymbol{r}_{0}\right)$, respectively. 


\section{References}

[1] Stein S 1961 Q. Appl. Math. 19 15-24

[2] Cruzan O R 1962 Q. Appl. Math. $2033-40$

[3] Auger J C, Stout B, Barrera R G and Curiel F 2001 J. Quant. Spectrosc. Radiat. Transfer 70 675-95

[4] Stout B, Auger J C and Lafait J 2001 J. Mod. Opt. 48 2105-28

[5] Stout B, Auger J C and Lafait J 2002 J. Mod. Opt. at press

[6] Mackowski D W 1994 J. Opt. Soc. Am. A 11 2851-61

[7] Mackowski D W and Miscshenko M I 1996 J. Opt. Soc. Am. $132266-78$

[8] Tayeb G and Maystre D 1997 J. Opt. Soc. Am. A 143323

[9] Gralak B, Enoch S and Tayeb G 2000 J. Opt. Soc. Am. A 17 1012
[10] Mackowski D W 1991 Proc. R. Soc. A 433 599-614

[11] Chew W C 1994 Waves and Fields in Inhomogeneous Media (IEEE Press Series on Electromagnetic Waves) (New York: IEEE Press)

[12] Siqueira P R and Sarabandi K 2000 IEEE Trans. Antennas Propag. 48

[13] Tzeng Y C and Fung A K 1994 J. Electr. Waves Appl. 8

[14] Leung Tsang, Jin Au Kong and Shin R T 1985 Theory of Microwave Remote Sensing (Wiley Series in Remote Sensing) (New York: Wiley)

[15] Mie G 1908 Ann. Phys., Lpz. 25 377-442

[16] Bohren C F and Huffman D R 1983 Absorption and Scattering of Light by Small Particles (New York: Wiley-Interscience) 